\title{
A Systematic Review of Recreation Therapy for Depression in Older Adults
}

\author{
Michael HS Lam ${ }^{1-4 *}$, Bik CHOW ${ }^{5}$, Siu Yin Cheung ${ }^{5}$, Ka Yiu Lee ${ }^{1-4}$, William Ho Cheung $\mathrm{Li}^{4}$, Eva Ho ${ }^{4}$, Stuart W Flint ${ }^{6}$, L Yang ${ }^{7}$ and Nathan Kin \\ Fai Yung ${ }^{8}$
}

${ }^{1}$ Academy of Sport and Physical Activity, Sheffield Hallam University, UK

${ }^{2}$ Hong Kong Sport Education and Leisure Tourism Foundation, HK

${ }^{3}$ International Industry and Professional Accreditation Association, HK

${ }^{4} \mathrm{Li}$ Ka Shing Faculty of Medicine, the University of Hong Kong, HK

${ }^{5}$ Department of Physical Education, Hong Kong Baptist University, HK

${ }^{6}$ Leeds Beckett University, UK

${ }^{7}$ Institute of Physical Education, University of Jinan, Shandong, China

${ }^{8}$ Asian Sport Psychology Professional Alliance, HK

\begin{abstract}
Background: Recreation therapy (RT) provides a flexible and powerful treatment for depression associated with aging. This article reviewed the effectiveness of RT to treat depression in older adults.

Method: Five electronic databases were employed to identify interventional studies on RT in depressed older adults: Pubmed, PsycINFO, ProQuest, Academic Search Premier and ERIC. Articles were screened against inclusion criteria and assessed with respect to methodological quality.
\end{abstract}

Results: A systematic literature review included 18 articles. Fourteen studies reported improvement in depression but 6 studies lack adequate significance in the positive effect of RT. Methodological quality assessment of 13 randomizedcontrolled trials and 5 non-controlled studies indicated an overall mean of $5.67 \pm 1.94$ points out of 9 .

Conclusion: There were positive findings that RT is effective in improving geriatric depression. Future investigation is encouraged to explore the mechanism between physical activity RT and depression improvement.

Keywords: Recreation therapy; Older adults; Depression; Systematic review

\section{Introduction}

Depression is among the most prevalent mental health condition affecting the elderly population [1-4]. Not only when quality of life (QOL) is most affected in elderly ladies, but the highest suicidal rate in US belongs to Caucasian men over 70 years old [5-7]. Simultaneously, recent evidence suggested that depressed mood was caused by more negative emotion input with less suitable conflict-solving strategies, and in turn it directly influenced the morbidity and mortality of chronic health conditions. Medical health conditions such as diabetes, stroke, cancers, and dementia became the biggest sources of stress $[8,9]$.

Psychosocially, isolation and unexpected changes in environment could be especially relevant to depressed mood. Henderson [10] found that married couples often share recreation and activity with each other. Loss of spouse would then discourage widows to continue recreational activities, which further limit the positive enjoyment for widows at old age. The loss of mobility further decreases self-esteem to carry out recreational activities $[11,12]$. As a stress-buffer and response to coping with stress, recreation is vital to the well-being of elderly people [13]. In addition, the length of institutionalization in older adults is positively associated with likelihood of depression and negatively associated with their self-esteem. In one study, $13 \%$ of residents develop one major depression over first year of institutionalization and 18\% develop new depressive symptoms [14].

Evidence showed that as low as one-tenth of depressed older adults received treatment for their depressed emotion [15]. The common barrier is that some symptoms such as changes in sleep, appetite, fatigue and loss of interests in usual activities can be overlooked as a part of aging. There is desperate need to enhance treatment for depression among elderly.
National Institute of Health [16] suggested that older adults prefer recreation therapy over medical treatment to depression. Recreation therapy (RT), also known as therapeutic recreation and recreational therapy, is defined by the National Therapeutic Recreation Society [17] in the US as "the therapeutic use of recreational methods in ways that facilitate enhancement of health, functional ability, independence, and perception of QOL. This is in line with one of the goals to increase QOL during treatment of depression". Especially for the elderly, recreation therapy has been proved to be effective to promote a healthy lifestyle, improve social life, release stress and depressed mood and with a potential to further shift their focus to obtain a better Quality of Life [18].

An advantage of RT is that such non-pharmacological intervention will not interfere with medication. Aged people are more likely to accept the medication treatment for their chronic conditions. The dynamic and diverse nature of RT can be more flexibly adjusted to suit the different physical and mental needs of depressed older adults.

Importantly, RT can address the social needs of older adults. A quote from the Bureau of Labor Statistics [19] illustrates the spectrum of activities under RT: "They use a variety of activities, including arts and crafts, drama, music, dance, sports, games and field trips. These activates

*Corresponding author: Michael Huen Sum LAM, 3/F Admin Block, 30 Shing Tai Road, Chai Wan, Hong Kong, Tel: 2595 2612; E-mail: michaelhslam@vtc.edu.hk

Received April 20, 2017; Accepted April 24, 2017; Published April 30, 2017

Citation: Lam MHS, Bik CHOW, Cheung SY, Lee KY, Li WHC, et al. (2017) A Systematic Review of Recreation Therapy for Depression in Older Adults. J Psychol Psychother 7: 298. doi: 10.4172/2161-0487.1000298

Copyright: @ 2017 Lam MHS, et al. This is an open-access article distributed unde the terms of the Creative Commons Attribution License, which permits unrestricted use, distribution, and reproduction in any medium, provided the original author and source are credited. 
help maintain or improve a client's physical and emotional well-being". RT encourages users to release any frustration from their circumstances and communicate with other people. For example, Jordan [20] observed that not only does RT improve Quality of Life; it also refreshes or encourages new purpose of life.

Research has identified the benefits when older adults took part in RT [21,22]. Creative activities nurture the sense of competence and capabilities, which assist older adults to adjust to the physically, psychologically or psychosocially changes around them.

Although overwhelming evidences supported that recreation therapy can benefit elderly to relieve their depression, there is not yet a guideline to allocate appropriate recreational intervention to any group of elderly people. Hunter and Gillen [9] suggested that designing stresscoping RT for elderly adults is challenging and yet well understood. Johnson [12] summarized the general relationship between RT and depression in elderly, which can be regarded as informative, but less systematic in the summary of the results. This lack of knowledge has motivated the current systematic review to update on the recent RT interventions available to the treatment of depression in elderly. The present article reviews the effectiveness of recreation therapy (RT) to treat depression in older adults.

\section{Method}

This review searched Pubmed, PsycINFO, ProQuest Academic Search Premier and ERIC, for interventional studies for geriatric depression. The alternative terms for the older age groups were based on a systematic review on health literacy in older people [23]. The following search strategy was used:

- ("elder*” OR "aged" OR "ageing" OR "older adults" OR "old"” OR "older people" OR "geriatr*” OR "senior" OR "nursing home")

- AND ("depression" OR "dysthymia" OR "depressive symptoms" OR "depressive disorder" OR "major depression")

- AND ("recreation therapy" OR "therapeutics" OR "recreational therapy" OR "therapeutic recreation")

- AND ("therapy" OR "treatment" OR "clinical trial" OR "psychosocial intervention" OR "nonpharmacological intervention" OR "outcome study" OR "randomized controlled trials")

Additionally, hand searches were performed by secondary scanning the references of eligible articles to include relevant studies.

\section{Inclusion criteria}

The final inclusion of articles had to fulfill all the following criteria:

1. Interventional study aimed to or included evaluation of depressive symptoms after RT.

2. Participants must be labeled as old according to the investigators. The World Health Organisation [24] defines old age as 60 years old or above, whereas others may use cut-off at age 65 .

3. Articles have to be in English and available in full text.

\section{Screening the studies}

The two of the authors of this paper worked in parallel stages. First, titles of search results are screened for potential eligibility. Second, the abstracts would be screened. Finally the full text of articles from search result will be assessed. Those that did not meet inclusion criteria were dropped out. Any contrasting decision was discussed until consensus was reached.

\section{Quality of included studies}

According to the methodological evaluation by Higgins and Green [25], the two authors carried out the assessment independently and discussed the results until consensuses were made. The mean scores and standard errors of each reviewed study were computed [26].

\section{Results}

Preliminary search results generated 694 articles. After the first round of screening excluded those incompatible with inclusion criteria, 305 articles remained. From there, 32 articles were left upon abstract screening against the inclusion criteria. During the final stage of full text screening, 15 studies remained from the electronic search. The review of reference lists added 3 more studies by hand searching. In conclusion, a total of 18 interventional studies were selected for use in present review.

The resulting inclusion contains 13 randomised-controlled trials (RCT) studies [18,27-38]. In addition, other 5 studies are non-controlled one-group interventions [39-43]. Table 1 summarizes the characteristics and design of the included studies.

In general, 1691 aged people were covered among eighteen studies while most of them were female and above 65 years old. Sample size ranged from 9 people to 439 people. All participants from eight studies were all diagnosed patients with depression [18,27,31,32,34,37,42], while older adults in another eight studies did not meet criteria for depression, instead they either suffered from chronic physical illness $[35,40]$, balance-problem $[29,38]$, dementia [36] and cognitive decline $[44,45]$ or were regular attendees $[39,43]$. The last two studies $[28,41]$ mixed up the depressed and non-depressed participants, in which the depressed elderly accounted for $48.3 \%$ and $52 \%$ of total participants respectively.

In consideration of the types of recreation therapy, the included studies can be divided into twelve exercise interventions, five psychosocial interventions and two combined exercise and psychosocial interventions. Physical interventions embraced multiple forms and included gardening [39], biking [18,27], balance training [29,38], Taichi and stretching [30], aerobic and resistance exercise [32,33,43] and Qigong [34,35]. Recreation therapy focusing on the psychological training comprised therapeutic intervention of anticipatory grief [40], support group [31], group reminiscence [36] and behavioral activation [42]. In particular, cognitive behavioral therapy was also regarded as a purely psychological intervention to treat for depressed elderly [37], since the content did not cover any behavioral activities. Besides, Suzanne Fitzsimmons and Buettner [28] combined 73 different types of recreation therapy, including wheelchair biking, relaxation, exercise and therapeutic cooking, which tailored to their needs for the participants. Similarly, Lai et al. [41] divided their intervention in three sessions including education, exercise and social support to connect physical and psychological health to social impact.

None of the 18 included studies reach the 9 point ideal methodological quality in Table 2 [25]. The scores range from 2 points [39] to 8 points $[29,30,33,34]$. Table 2 illustrates the assessment of the included articles. The overall mean score is $5.67 \pm 1.94$ points. The randomized control trail $(\mathrm{RCT})$ studies have a higher $($ mean $=6.54 \pm 1.39)$ than the non-RCT interventional studies (mean $=3.40 \pm 1.14)$. 
Citation: Lam MHS, Bik CHOW, Cheung SY, Lee KY, Li WHC, et al. (2017) A Systematic Review of Recreation Therapy for Depression in Older Adults. J Psychol Psychother 7: 298. doi: 10.4172/2161-0487.1000298

Page 3 of 7

\begin{tabular}{|c|c|c|c|c|c|}
\hline Author & Interventional and Comparison & Study Design/Length & $\mathbf{N}$ & $\begin{array}{l}\text { Outcome } \\
\text { Measures }\end{array}$ & Significant Effects * \\
\hline \multirow[t]{2}{*}{ Austin et al. [39] } & $\begin{array}{l}\text { T: Planned gardening activities with } \\
\text { specific health goals }\end{array}$ & One-group pre-/post-test & $\begin{array}{l}\mathrm{T}=6 \\
\text { Age }=68\end{array}$ & $\begin{array}{l}\text { Total Emotional } \\
\text { Score in COOP; } \\
\text { GDS }\end{array}$ & $\begin{array}{l}\text { Total Emotional Score }(p=0.042) \\
\text { GDS score changed without statistical } \\
\text { significance }\end{array}$ \\
\hline & & 8 weeks & & & \\
\hline \multirow[t]{2}{*}{$\begin{array}{l}\text { Buettner and } \\
\text { Fitzsimmons [28] }\end{array}$} & $\begin{array}{l}\text { T: Intensive wheelchair AD-biking and } \\
\text { maintenance intervention period. }\end{array}$ & $\mathrm{RCT}$ & $\mathrm{T}=41$ & $\begin{array}{l}\text { GDS; MMSE; } \\
\text { CMAI }\end{array}$ & $\begin{array}{l}\text { GDS within treatment group at post-test } \\
(p<0.001) \text {; within treatment group at follow- } \\
\text { up }(p<0.001) \text {; between group at post-test } \\
(p=0.047)\end{array}$ \\
\hline & C: Not mentioned & $\begin{array}{l}2 \text { weeks of } 5 \text { days daily } \\
1 \text { hour discussion and } \\
\text { riding session; } 10 \text {-week } \\
\text { maintenance included two } \\
\text { rides per week }\end{array}$ & $C=29$ & & \\
\hline \multirow[t]{2}{*}{ Cheng et al. [40] } & $\begin{array}{l}\text { T: Anticipatory grief therapy with } \\
\text { experiential and expressive activities }\end{array}$ & $\begin{array}{l}\text { One-group pre-/post-test } \\
\text { and follow-up }\end{array}$ & $\begin{array}{l}T=24 \\
\text { Age }=81.8\end{array}$ & $\begin{array}{l}\text { GDS-Chinese; } \\
\text { MQOL-HK } \\
\text { (psychological } \\
\text { score) }\end{array}$ & $\begin{array}{l}\text { GDS-Chinese decreased significantly } \\
\text { at post-test }(p=0.013) \text {, but not at follow- } \\
\text { up; MQOL-HK psychological measure } \\
\text { decreased at post-test }(p=0.027) \text {, but not at } \\
\text { follow-up }\end{array}$ \\
\hline & & $\begin{array}{l}4 \text { weekly } 1.5 \mathrm{~h} \text { sessions, } 4 \\
\text { week follow up }\end{array}$ & & & \\
\hline \multirow[t]{2}{*}{ Fitzsimmons [18] } & $\begin{array}{l}\text { T: intensive Easy-rider therapy with } \\
\text { small group discussion and 15-min } \\
\text { bike ride }\end{array}$ & RCT & $\begin{array}{l}T=19 \\
\text { Age }=80.8\end{array}$ & GDS & $\begin{array}{l}\text { GDS improvement in treatment group at } \\
\text { post-test }(p<0.001)\end{array}$ \\
\hline & $\begin{array}{l}\text { C: Did not receive the therapy biking } \\
\text { program until after post-test }\end{array}$ & $\begin{array}{l}2 \text { weeks of } 5 \text { daily } 1 \mathrm{~h} \\
\text { session }\end{array}$ & $\begin{array}{l}\mathrm{C}=20 \\
\mathrm{Age}=80.2\end{array}$ & & \\
\hline \multirow[t]{2}{*}{$\begin{array}{l}\text { Buettner and } \\
\text { Fitzsimmons [28] }\end{array}$} & $\begin{array}{l}\text { T: individually prescribed therapeutic } \\
\text { intervention in small groups }\end{array}$ & RCT. & $\begin{array}{l}T=29 \\
\text { Age }=81.3\end{array}$ & $\begin{array}{l}\text { CMAI; BFS; } \\
\text { PiDS; BVP; HR }\end{array}$ & $\begin{array}{l}\text { CMAI improvement at post-test }(p=0.029) ; \\
\text { PiDS improvement at post-test }(p<0.001) ; \\
\text { positive correlation between PiDS and BVP } \\
(p=0.013) ; \text { positive correlation between } \\
\text { agitation and HR }(p=0.018)\end{array}$ \\
\hline & $\begin{array}{l}\text { C: Usual home care for two weeks } \\
\text { before delayed intervention }\end{array}$ & $\begin{array}{l}2 \text { weeks of } 3-5 \text { daily } \\
\text { session of } 1.5 \mathrm{~h}\end{array}$ & $C=30$ & & \\
\hline \multirow[t]{2}{*}{ Halvarsson et al. [29] } & T: Progressive group balance training & RCT & $\mathrm{T}=38$ & $\begin{array}{l}\text { FESI; GDS-20; } \\
\text { perceived fear } \\
\text { of falling }\end{array}$ & $\begin{array}{l}\text { FESI score in treatment group continuously } \\
\text { decreased throughout } 15 \text { month follow } \\
\text { up }(p=0.001) \text {; GDS did not changed } \\
\text { significantly }\end{array}$ \\
\hline & C: Not mentioned & $\begin{array}{l}345-\text { min sessions per } \\
\text { week for } 12 \text { weeks. } 15 \\
\text { month follow up. }\end{array}$ & $C=21$ & & \\
\hline \multirow[t]{2}{*}{ Lai et al. [41] } & $\begin{array}{l}\text { T: video conference intervention of } \\
\text { education, strength and balance } \\
\text { exercise and social support }\end{array}$ & One-group pre-/post-test. & $\begin{array}{l}T=19 \\
\text { Age }=69.5\end{array}$ & $\begin{array}{l}\text { BBS; SSES; } \\
\text { SF-36; GDS }\end{array}$ & $\begin{array}{l}\text { After intervention, scores SSES, BBS and } \\
\text { SF-36 improved }(p<0.001)\end{array}$ \\
\hline & & $\begin{array}{l}8 \text { weeks of } 1.5 \mathrm{~h} \text { session } \\
\text { per week with education, } \\
\text { exercise, social support } \\
\text { training }\end{array}$ & & & \\
\hline \multirow[t]{2}{*}{ Lam et al. [30] } & $\begin{array}{l}\text { T: } 1 \text { year program to practice } 24 \\
\text { simplified forms for Tai Chi }\end{array}$ & RCT & $\begin{array}{l}\mathrm{T}=171 \\
\text { Age }=77.2\end{array}$ & CDS; & $\begin{array}{l}\text { The Cornell Depression Score did not } \\
\text { decreased significantly after intervention }\end{array}$ \\
\hline & $\begin{array}{l}\text { C: } 1 \text { year of stretching and muscle } \\
\text { toning exercise }\end{array}$ & $\begin{array}{l}\text { At least } 30 \text { min per day, at } \\
\text { least } 3 \text { times a week for } \\
1 \text { year }\end{array}$ & $\begin{array}{l}C=218 \\
\text { Age }=78.3\end{array}$ & & \\
\hline \multirow[t]{2}{*}{ Ong et al. [31] } & $\begin{array}{l}\text { T: Support group for recently discharged } \\
\text { depressed patients by psychogeriatric } \\
\text { social worker and community } \\
\text { psychiatric nurse }\end{array}$ & RCT & $\begin{array}{l}T=10 \\
\text { Age }=72.8\end{array}$ & $\begin{array}{l}\text { CAPE; ZDI; re- } \\
\text { referral rate; re- } \\
\text { admission rate }\end{array}$ & $\begin{array}{l}\text { Depression rate did not have any significant } \\
\text { changes }\end{array}$ \\
\hline & C: No support group & 1 year & $\begin{array}{l}C=10 \\
\text { Age }=76.7\end{array}$ & & \\
\hline Penninx et al. [32] & $\begin{array}{l}\text { T1: supervised facility-based and } \\
\text { independent home-based walking } \\
\text { program }\end{array}$ & $\mathrm{RCT}$ & $\mathrm{T} 1=149$ & CES-D & $\begin{array}{l}\text { CES-D score dropped in overall T1 } \\
(p=0.004) \text {, in the low depressive } \\
\text { symptomatology group }(p=0.01) \text { and high } \\
\text { depressive symptomatology group }(p=0.03)\end{array}$ \\
\hline
\end{tabular}


Citation: Lam MHS, Bik CHOW, Cheung SY, Lee KY, Li WHC, et al. (2017) A Systematic Review of Recreation Therapy for Depression in Older Adults. J Psychol Psychother 7: 298. doi: 10.4172/2161-0487.1000298

Page 4 of 7

\begin{tabular}{|c|c|c|c|c|c|}
\hline & $\begin{array}{l}\text { T2: supervised upper and lower body } \\
\text { weight training }\end{array}$ & $\begin{array}{l}\text { Three } 1 \mathrm{~h} \text { session per } \\
\text { week over } 3 \text { months }\end{array}$ & $\mathrm{T} 2=146$ & & \\
\hline & $\begin{array}{l}\text { C: Health education by nurse on } \\
\text { arthritis management }\end{array}$ & & $C=144$ & & \\
\hline \multirow[t]{2}{*}{ Singh et al. [33] } & $\begin{array}{l}\text { T: supervised weight-lifting exercise and } \\
\text { unsupervised exercises }\end{array}$ & $\mathrm{RCT}$ & $\mathrm{T}=17$ & BDI & $\begin{array}{l}\text { BDI scores remained lower in the exercise } \\
\text { group at } 20 \text { th and } 26 \text { th week ( } p=0.036 \\
\text { and } p=0.047) \text { compared with controls; BDI } \\
\text { continued to improve over time (time } \times \\
\text { treatment interaction } p=0.001)\end{array}$ \\
\hline & C: health education lectures & 20 weeks & $C=15$ & & \\
\hline \multirow[t]{2}{*}{ Tsang et al. [35] } & T: Supervised Qigong session & RCT & $\mathrm{T}=24$ & GDS-Chinese & No significant decline in GDS measure \\
\hline & $\begin{array}{l}\text { C: traditional remedial rehabilitation } \\
\text { activities }\end{array}$ & $\begin{array}{l}\text { Two } 1 \mathrm{~h} \text { sessions per week } \\
\text { for } 12 \text { weeks }\end{array}$ & $C=26$ & & \\
\hline \multirow[t]{2}{*}{ Tsang et al. [34] } & T: Supervised Qigong session & RCT. & $\mathrm{T}=48$ & GDS-Chinese; & $\begin{array}{l}\text { Treatment group showed better scores } \\
\text { in GDS at time by group }(p=0.041) \text {, over- } \\
\text { time }(p<0.01) \text {, group differences }(p<0.01) \\
\text { interaction, but not significantly in follow-up } \\
\text { sessions }\end{array}$ \\
\hline & $\begin{array}{l}\text { C: newspaper reading group with } \\
\text { qualified therapist }\end{array}$ & $\begin{array}{l}\text { Three } 30-45 \text { min session } \\
\text { per week over } 16 \text { weeks }\end{array}$ & $C=34$ & & \\
\hline \multirow[t]{2}{*}{ Wang [36] } & T: Group reminiscence therapy & RCT & $\begin{array}{l}T=51 \\
\text { Age }=79.8\end{array}$ & GDS, CSDD & $\begin{array}{l}\text { Treatment group showed better scores in } \\
\text { CSDD }(p=0.026) \text { and slight improvement } \\
\text { in GDS }\end{array}$ \\
\hline & C: Not Mentioned & $\begin{array}{l}1 \mathrm{~h} \text { weekly sessions over } \\
8 \text { weeks }\end{array}$ & $\begin{array}{l}\mathrm{C}=51 \\
\text { Age }=78.9\end{array}$ & & \\
\hline \multirow[t]{2}{*}{ Wilkinson et al. [37] } & $\begin{array}{l}\text { T: Antidepressant medical treatment } \\
\text { and CBT }\end{array}$ & RCT & $\begin{array}{l}\mathrm{T}=22 \\
\text { Age }=72.7\end{array}$ & $\begin{array}{l}\text { Frequency of } \\
\text { recurrence at } 6 \\
\text { and } 12 \text { month; } \\
\text { BDI; MADRS }\end{array}$ & $\begin{array}{l}\text { MADRS decreased without significant. } \\
\text { BDI score slightly increased. }\end{array}$ \\
\hline & $\begin{array}{l}\text { C: Antidepressant medical treatment } \\
\text { alone. }\end{array}$ & $\begin{array}{l}2.5 \text { months of CBT, } 12 \\
\text { month of antidepressant }\end{array}$ & $\begin{array}{l}C=23 \\
\text { Age }=75.2\end{array}$ & & \\
\hline \multirow[t]{2}{*}{ Wolf et al. [38] } & T: Short, individualized balance training & $\mathrm{RCT}$ & $\begin{array}{l}\mathrm{T}=47 \\
\text { Age }=84.5\end{array}$ & HADS & HADS did not changed significantly \\
\hline & $\begin{array}{l}\text { C: Individualized extra attention } \\
\text { program }\end{array}$ & $\begin{array}{l}\text { 4-6 weeks exercise in } \\
\text { sitting, standing and } \\
\text { walking }\end{array}$ & $\begin{array}{l}C=47 \\
\text { Age }=83.6\end{array}$ & & \\
\hline \multirow[t]{2}{*}{ Yon and Scogin [42] } & $\begin{array}{l}\text { T: Behavioral activation therapy with } \\
\text { psychologists }\end{array}$ & One-group pre-/post-test & $\begin{array}{l}T=9 \\
\text { Age }=75\end{array}$ & $\begin{array}{l}\text { GDS-30, } \\
\text { HRSD-17, DAS, } \\
\text { PES, GSHR }\end{array}$ & $\begin{array}{l}\text { GDS score was reduced after intervention } \\
(p<0.05)\end{array}$ \\
\hline & & $\begin{array}{l}\text { Twice } 1 \mathrm{~h} \text { session per } \\
\text { week over first } 4 \text { months, } \\
\text { once weekly afterwards }\end{array}$ & & & \\
\hline \multirow[t]{3}{*}{ Yu et al. [43] } & T1: Relaxation and tensing of muscles & 3 groups pre-test post-test & $\begin{array}{l}\text { T1 }=59 \\
\text { Age }=74.9\end{array}$ & HADS; & $\begin{array}{l}\text { Comparing with control group, the relaxation } \\
\text { group reduced psychological distress } \\
\text { in the HADS }(p=0.001) \text {, of which both } \\
\text { anxiety and depression subscales were } \\
\text { affected ( } p=0.017 \text { and } p=0.033 \text { ). Whereas } \\
\text { the exercise training could only reduce } \\
\text { the anxiety subscale of HADS ( } p=0.033 \text { ). } \\
\text { Significant Group X time interactions were } \\
\text { observed in all HADS subscales (total score } \\
p=0.002 \text {; anxiety score } p=0.007 \text {; depression } \\
\text { score } p=0.009 \text { ). }\end{array}$ \\
\hline & $\begin{array}{l}\text { T2: exercise therapy including strength, } \\
\text { resistance training and aerobic dance }\end{array}$ & 12 weeks & $\begin{array}{l}\text { T2 }=32 \\
\text { Age }=73\end{array}$ & & \\
\hline & $\begin{array}{l}\mathrm{C}=\text { received attention through } \\
\text { telephone calls by research nurse }\end{array}$ & & $\begin{array}{l}\text { C }=62 \\
\text { Age }=77.6\end{array}$ & & \\
\hline
\end{tabular}


6MWT=6 Minute Walk Test; ADAS-Cog=The Cantonese Version of Alzheimer's Disease Assessment Scale - Cognitive Subscale; ASSEI=Self-Concept Scale; BBS=Berg Balance Scale; BDI=Beck Depression Inventory; BFS=Behavior Flow Sheet; BVP=Blood Volume Pulse; C=Control Group; CBT-G=Group Cognitive Behaviour Therapy; $\mathrm{CBT}=$ Cognitive Behavioral Therapy; CDS=Cornell Depression Score; CES-D=Centre for Epidemiologic Studies Depression Scale; CGSS=Chinese General Self-Efficacy Scale; CHQ-C=Chronic Heart Failure Questionnaire CMAI=Cohen-Mansfield Agitation Inventory; CMMSE=Cantonese Version of Mini-Mental State Examination; COOP Charts=The Dartmouth COOP Functional Health Assessment Charts; CSDD=Cornell Scale for Depression in Dementia; DAS=Dysfunctional Attitudes Scale; DGI=Dynamic Gait Index; FESI=Fall Efficacy Scale-International; GDS-20=Geriatric Depression Scale - 20 items; GDS-30=Geriatric Depression Scale - 30 Item; GDS-Chinese=Geriatric Depression Scale 15-item Chinese Version; GDS=Geriatric Depression Scale Short Form; GHQ-12=General Health Questionnaire-12; GSHR=Global Status Health Rating; HR=Heart Rate; HRSD-17=17-Item Hamilton Rating Scale for Depression; MADRS=Montgomery Asberg Depression Rating Scale; MMSE=Mini-Mental State Examination; MOS-SSS=The Medical Outcomes Study Social Support Survey; MQOL-HK=McGill Quality of Ling Questionnaire-Hong Kong version; PBQ=Perceived Benefit Questionnaire; PES=Pleasant Events Scale; PiDS=Passivity in Dementia Scale; PWI=Personal Well Being Index; QOL=Quality of Life; SF-36=Medical Outcomes Study 36-Item Short Form; SSES=State Self-Esteem Scale; T=Treatment Group; WHOQOL-BREF[HK]=Hong Kong Chinese Version World Health Organization Quality of Life: Abbreviated Version

Table 1: Characteristics and findings of included studies.

\begin{tabular}{|c|c|c|c|c|c|c|c|c|c|c|}
\hline Included Studies & A & B & C & D & $\mathbf{E}$ & $\mathbf{F}$ & G & $\mathbf{H}$ & $\mathbf{I}$ & TOTAL \\
\hline Austin et al. [39] & - & - & + & $\mathrm{u}$ & - & - & - & - & + & 2 \\
\hline Buettner and Fitzsimmons [28] & + & + & + & + & - & u & - & - & + & 5 \\
\hline Cheng et al. [40] & - & - & + & $\mathrm{u}$ & - & + & - & - & + & 3 \\
\hline Fitzsimmons [18] & + & - & + & + & - & + & - & - & + & 5 \\
\hline Buettner and Fitzsimmons [28] & + & + & + & + & - & + & - & - & + & 5 \\
\hline Halvarsson et al. [29] & + & + & + & + & + & + & - & + & + & 8 \\
\hline Lai et al. [41] & - & - & + & $\mathrm{u}$ & - & + & - & - & + & 3 \\
\hline Lam et al. [23] & + & + & + & + & + & + & - & + & + & 8 \\
\hline Ong et al. [31] & + & + & + & + & + & + & - & - & + & 7 \\
\hline Penninx et al. [32] & + & + & + & + & + & + & - & - & + & 7 \\
\hline Singh et al. [33] & + & + & + & + & + & + & - & + & + & 8 \\
\hline Tsang et al. [35] & + & + & + & + & - & + & - & + & + & 7 \\
\hline Tsang et al. [34] & + & + & + & + & + & + & - & + & + & 8 \\
\hline Wang [36] & + & + & + & + & - & + & - & - & + & 6 \\
\hline Wilkinson et al. [37] & + & - & + & + & - & - & - & - & + & 4 \\
\hline Wolf et al. [38] & + & + & + & + & - & + & - & + & + & 7 \\
\hline Yon and Scogin [42] & - & - & + & - & - & + & - & + & + & 4 \\
\hline Yu et al. [43] & - & - & + & + & + & + & - & - & + & 5 \\
\hline \multicolumn{11}{|c|}{$\begin{array}{l}\text { Quality criteria: } A=\text { Randomization, } B=A \text { llocation concealment, } C=\text { Inclusion/exclusion criteria specified, } D=S \text { Similarity of groups at baseline, } E=A s s e s s o r s \text { blinded to } \\
\text { outcome, } F=\text { Characteristics of participants lost to follow-up described, } G=\text { Intention-to-treat analysis, } H=\text { Power calculated, I=Outcome measures valid }\end{array}$} \\
\hline \multicolumn{11}{|c|}{$+=$ Criterion fulfilled, $-=$ Criterion not fulfilled, $u=$ Unknown if criterion is fulfilled } \\
\hline
\end{tabular}

Table 2: Quality assessment and total score of the included studies

\section{Discussion}

This systematic review included eighteen investigations to different RT programs between 1987 and 2013. Fourteen studies reported depressive symptom improvement after intervention, of which six studies did not have sufficient significance at alpha $=0.05$ $[29,30,31,35,38,39]$. The general findings provide evidence to support the effectiveness of RT in the treatment of geriatric depression.

Although 11 of 14 depression-improved interventions focused on physical activity, it should be noted that the intensity of physical activity involved does not seem to play an important role in the process. For example, participants in the study by Buettner and Fitzsimmons [27] were pushed around on a bike. The intensity of this bike activity was feeble when compared to Qi-gong practice in Tsang et al. [35], which had no significant results. It may be argued that the differences in sample size, intervention frequency and length could have affected the outcomes. Further evidence from the 3-group pretest post-test study [43] suggested that, in the relaxation training and exercise training intervention programs, participants reported different levels of improvement on the Hospital Anxiety and Depression Scale (HADS) subscales. Although both interventions had improvement in depression, only the relaxation group yielded significance from the HADS depression subscale while exercise group did not. The differences between the results with other effective exercise training may be due to non-randomized samplings and variations on age and sample size. The observation of negative interaction between physical activity intensity and improvement to depression from this review encourages future investigations.

Likewise, the psychological training in recreational therapy cannot ensure a stable and obvious result. Three interventions were effective with two showing significant changes $[40,42]$ and one with a slight positive change [31]. Moreover, Wang [36] applied Cornell Scale for Depression in Dementia and Geriatric Depression Scale short form to evaluate the changes, and after intervention the former one has remarkable improvement relative to the minimal changes for the latter. Another divergence has been presented in the study by Wilkinson et al. [37], which showed that increasing depression degree in Beck Depression Inventory (BDI) was not consistent with the slight changes occurred in scores of Montgomery Asberg Rating Scale for Depression after training. Wilkinson et al. [37] suggested the BDI was not suitable for studies with depressed elderly. This invites further investigations.

Unfortunately, the only two interventions that combined physical and psychological types of recreation therapy cannot offer clear conclusion in the context of this review. Fitzsimmons and Buettner [28] focused more on passivity and agitation on elderly with dementia, rather than depression degree. Lai et al. [41] only assessed the depressed symptoms at baseline, but did not demonstrate the changes after 
intervention. Theoretically, such combined interventions can draw on the strengths of different forms of recreation therapy and provide the greatest benefits for depressed elderly. Further efforts should be done to fill in the research blanks.

In view of whether the study is helpful in designing therapy for depressed old persons, six of eight studies with pure depressed participants showed significant improvement, while five of eight studies with non-depressed participants only had encouraging but statistically insignificant changes, which can lead to a conclusion that recreational therapy can effectively moderate depression for most elderly, especially for those who displayed depressive symptoms.

Methodological limitations exist in the included studies. Universally, none of the studies fully addressed the intention-to-treat analysis. In the intervention studies, many older adults with high risk of health problems encountered high frequency of attrition. For example, Tsang et al. [35] utilized the RCT design but found no significant result after treatment. An intention-to-treat analysis could help to avoid bias resulting from drop out and crossover effect. Assessments of reviewed articles relied on two independent authors who provided subjective judgments. It is important to have objective assessments [46-51] to improve the objectivity. Meta-analyses and other in-depth statistical analyses $[52,53]$ were not conducted due to the nature of this study which may affect the findings [54].

The variety of intervention portrayed by the included studies fully demonstrated how flexible and accommodating RT can be applied to treat depression in older adults. From gardening [39] to weight-lifting [33], from group reminiscence [36] to anticipatory grief therapy [40], for stroke patients [41] and for dementia patients [28], RT had wide applications in treating geriatric depression. However, the diversity of $\mathrm{RT}$ approaches so far used and reported in this analysis of the literature make it difficult to fully determine the cost-effectiveness of RT in the context of care-givers.

\section{Conclusion}

To sum up, this systematic review of intervention studies evaluating RT programs concludes that RT is an effective treatment to improve depression in older adults, albeit the aforementioned limitations. Although the benefits of physical activity [23,44,45,55-61] and sports [62-67] have been extensively examined, the effects of physical activity and sport RT on depression warrants further exploration.

\section{Acknowledgement}

We are grateful for the support of academic board members of Hong Kong Sport Education and Leisure Tourism foundation, Hong Kong.

\section{References}

1. Luppa M, Sikorski C, Luck T, Ehreke L, Konnopka A, et al. (2012) Age-and gender-specific prevalence of depression in latest-life-systematic review and meta-analysis. J Affect Disord 136: 212-221.

2. Miller DK, Coe RM, Romeis JC, Morley JE (1995) Improving quality of geriatric health care in four delivery sites: suggestions from practitioners and experts. $J$ Am Geriatr Soc 43: 60-65.

3. Rajkumar A, Thangadurai P, Senthilkumar P, Gayathri K, Prince M, et al. (2009) Nature, prevalence and factors associated with depression among the elderly in a rural south Indian community. Int Psychogeriatr 21: 372-378.

4. Wu Z, Schimmele CM, Chappell NL (2012) Aging and late-life depression. J Aging Health 24: 3-28.

5. Conwell Y, Rotenberg M, Caine ED (1990) Completed suicide at age 50 and over. J Am Geriatr Soc 38: 640-644.

6. Jörngården A, Wettergen L, von Essen L (2006) Measuring health-related quality of life in adolescents and young adults: Swedish normative data for the SF-36 and the HADS and the influence of age, gender and method of administration. Health Qual Life Outcomes 4: 91.

7. Sampogna F, Chren M, Melchi C, Pasquini P, Tabolli S, et al. (2006) Age, gender quality of life and psychological distress in patients hospitalized with psoriasis. Br J Dermatol 154: 325-331.

8. Boardman JD (2004) Stress and physical health: The role of neighborhoods as mediating and moderating mechanisms. Soc Sci Med 58: 2473-2483.

9. Hunter IR, Gillen MC (2009) Stress coping mechanisms in elderly adults: An initial study of recreational and other coping behaviors in nursing home patients. Adultspan Journal 8: 43-53.

10. Henderson KA (1996) One size doesn't fit all: The meanings of women's leisure $\mathrm{J}$ Leis Res 28: 139-154.

11. Csikszentmihalyi M (1997) Finding flow: The psychology of engagement with everyday life. Basic Books, New York.

12. Johnson CD (2000) Therapeutic recreation treats depression in the elderly. Home Health Care Serv Q 18: 79-90.

13. Trenberth $L$, Dewe $P(2002)$ The importance of leisure as a means of coping with work related stress: An exploratory study. Couns Psychol Q 15: 59-72.

14. Engedal K, Barca ML, Laks J, Selbaek G (2011) Depression in Alzheimer's disease: Specificity of depressive symptoms using three different clinical criteria. Int J Geriatr Psychiatr 26: 944-951.

15. Ell K (2006) Depression care for the elderly: reducing barriers to evidencebased practice. Home Health Care Serv Q 25: 115-148.

16. National Institute of Health (1991) Diagnosis and treatment of depression in late life: NIH consensus statement.

17. National Therapeutic Recreation Society (2000) Definition statement Ashburn VA National Recreation and Park Association.

18. Fitzsimmons $S$ (2001) Easy rider wheelchair biking. A nursing-recreation therapy clinical trial for the treatment of depression. J Gerontol Nurs 27: 14-23.

19. Bureau of Labor Statistics (U.S.D.L) (2012) Occupational outlook handbook, recreational therapists.

20. Jordan J (1998) Lecture from leisure and aging: Ulyssean living in later life Oklahoma State University, Leisure Studies Program, Stillwater, OK.

21. Fisher BJ, Specht DK (2000) Successful aging and creativity in later life. J Aging Stud 13: 457-472.

22. Flood M, Phillips KD (2007) Creativity in older adults: A plethora of possibilities Issues Ment Health Nurs 28: 389-411.

23. Lam MHS, Leung A, Chan S (2011) Psychological and cognitive determinants of the health literacy on soon-to-be-aged and older adults: A systematic review. Imanagers J Nurs 1: 46-56.

24. World Health Organisation (2013) WHO Fact Sheet No.344 Falls.

25. Higgins JP, Green S (2008) Cochrane handbook for systematic reviews of interventions. John Wiley \& Sons, Chichester UK.

26. Lee KY, Cerin E (2014) Standard Errors. In: AC Michalos (Ed.) Encyclopedia of Quality of Life and Well-Being Research. Springer, Netherlands.

27. Buettner LL, Fitzsimmons S (2005) Best practice in training and implementing evidence-based care for behaviors. Alzheimers Dementia 1: S55.

28. Fitzsimmons S, Buettner LL (2002) Therapeutic recreation interventions for need-driven dementia-compromised. Am J Alzheimers Dis Other Demen 17: 367-381.

29. Halvarsson A, Franzén E, Farén E, Olsson E, Oddsson L, et al. (2013) Longterm effects of new progressive group balance training for elderly people with increased risk of falling-a randomized controlled trial. Clin Rehabil 27: 450-458.

30. Lam LC, Chau R, Wong BM, Fung AW, Lui VW, et al. (2011) Interim follow $\square$ up of a randomized controlled trial comparing Chinese style mind body (Tai Chi) and stretching exercises on cognitive function in subjects at risk of progressive cognitive decline. Int J Geriatr Psychiatry 26: 733-740.

31. Ong YL, Martineau F, Lloyd C, Robbins I (1987) A support group for the depressed elderly. Int J Geriatr Psychiatry 2: 119-123. 
Citation: Lam MHS, Bik CHOW, Cheung SY, Lee KY, Li WHC, et al. (2017) A Systematic Review of Recreation Therapy for Depression in Older Adults. J Psychol Psychother 7: 298. doi: 10.4172/2161-0487.1000298

Page 7 of 7

32. Penninx BW, Rejeski WJ, Pandya J, Miller ME, Di Bari M, et al. (2002) Exercise and depressive symptoms a comparison of aerobic and resistance exercise effects on emotional and physical function in older persons with high and low depressive symptomatology. J Gerontol B Psychol Sci Soc Sci 57: 124-132.

33. Singh NA, Clements KM, Singh MAF (2001) The efficacy of exercise as a long-term antidepressant in elderly subjects a randomized, controlled trial. $\mathrm{J}$ Gerontol A Biol Sci Med Sci 56: 497-504.

34. Tsang HW, Fung KM, Chan AS, Lee G, Chan F (2006) Effect of a qigong exercise programme on elderly with depression. Int J Geriatr Psychiatry 21: 890-897

35. Tsang HW, Mok C, Au Yeung Y, Chan SY (2003) The effect of Qigong on general and psychosocial health of elderly with chronic physical illnesses: a randomized clinical trial. Int J Geriatr Psychiatry 18: 441-449.

36. Wang JJ (2007) Group reminiscence therapy for cognitive and affective function of demented elderly in Taiwan. Int J Geriatr Psychiatry 22: 1235-1240.

37. Wilkinson P, Alder N, Juszczak E, Matthews H, Merritt C, et al. (2009) A pilo randomised controlled trial of a brief cognitive behavioural group intervention to reduce recurrence rates in late life depression. Int J Geriatr Psychiatry 24: 68-75.

38. Wolf B, Feys H, De Weerdt W, van der Meer J, Noom M, et al. (2001) Effect of a physical therapeutic intervention for balance problems in the elderly: $A$ single-blind, randomized, controlled multicentre trial. Clin Rehabil 15: 624-636.

39. Austin EN, Johnston YA, Morgan LL (2006) Community gardening in a senior center: $A$ therapeutic intervention to improve the health of older adults. Therapeutic Recreation Journal 40: 48-57.

40. Cheng J, Lo R, Chan F, Woo J (2010) A pilot study on the effectiveness of anticipatory grief therapy for elderly facing the end of life. J Palliat Care 26 261-269.

41. Lai JC, Woo J, Hui E, Chan WM (2004) Telerehabilitation - a new model for community-based stroke rehabilitation. J Telemed Telecare 10: 199-205.

42. Yon A, Scogin F (2009) Behavioral activation as a treatment for geriatric depression. Clin Gerontol 32: 91-103.

43. Yu DS, Lee DT, Woo J, Hui E (2007) Non-pharmacological interventions in older people with heart failure: effects of exercise training and relaxation therapy. Gerontology 53: 74-81.

44. Lam MHS, Cheung SY, Chow BC (2011a) The effects of Tai-Chi-Soft-Ball training on physical functional health of Chinese older adult. Journal of Human Sport and Exercise 6: 540-553.

45. Lam MHS, Cheung SY, Chow BC (2011b) Effects of Tai Chi soft ball training on health-related quality of life of older adults with functional limitations. Asian Journal of Gerontology and Geriatrics 6: 65-71.

46. Cerin E, Chan KW, Macfarlane DJ, Lee KY, Lai PC (2011) Objective assessment of walking environments in ultra-dense cities: Development and reliability of the Environment in Asia Scan Tool-Hong Kong version (EAST-HK). Health Place 17: 937-945.

47. Cerin E, Lee KY, Barnett A, Sit CH, Cheung MC, et al. (2013) Objectivelymeasured neighborhood environments and leisure-time physical activity in Chinese urban elders. Prev Med 56: 86-89.

48. Lee KY, Lam MHS, Deng Y (2017b) Measuring post-concussive activity levels of patients: Step count or activity intensity? JAMA Pediatr.

49. Lee KY, Lam MHS, Lee PH (2017) Distance from home to the nearest tobacco outlet may not reflect the true accessibility. JAMA Int Med 177: 287-287.

50. Lee KY, Macfarlane D, Cerin E (2013a) Objective evaluation of recreationa facilities: Development and reliability of the recreational facility audit tool. J Park Recreat Admi 31: 92-109.

51. Lee KY, Macfarlane DJ, Cerin E (2013b) Comparison of three models of actigraph accelerometers during free living and controlled laboratory conditions. Eur J Sport Sci 13: 332-339.

Citation: Lam MHS, Bik CHOW, Cheung SY, Lee KY, Li WHC, et al. (2017) A Systematic Review of Recreation Therapy for Depression in Older Adults. J Psychol Psychother 7: 298. doi: 10.4172/2161-0487.1000298
52. Lee $\mathrm{PH}$, Tse AC, Lee KY (2016)A new statistical model for the day reconstruction method. Int J Methods Psychiatr Res.

53. Deng Y, Lee KY, Lam MHS, Lee PH (2016) Understanding sociobehaviora mitigators of depressive symptoms among US young adults. Behav Med 42 217-226.

54. Lee KY, Lam MHS, Deng Y (2017a) Interventions for anxiety and depression in conflict-affected areas. JAMA 317: 1376-1376

55. Cerin E, Lee KY, Barnett A, Sit CH, Cheung MC, et al. (2013) Walking for transportation in Hong Kong Chinese urban elders: a cross-sectional study on what destinations matter and when. Int J Behav Nutr Phys Act 10: 78

56. Fung L, Lam MHS (2012) Effectiveness of a progressive stepping program on lower limb function in community dwelling older adults. J Exerc Sci Fit 10: 8-11.

57. Lam MHS (2016) Exercise game exhilarates the elderly: A challenge to traditional training. J Athl Enhanc 5: 1.

58. Lam MHS, Kok EYL, Louie HTL, Lee KY (2014) External Chinese martial arts and health. In: S FONG (Ed.), Martial Arts for Health: Translating Research into Practice. OMICS Group Incorporation, USA, CA.

59. Lam MHS, Leung AYM (2016) The effectiveness of health literacy oriented programs on physical activity behaviour in middle aged and older adults with type 2 diabetes: A systematic review. Health Psychol Res 4: 5595

60. Lee KY, Lam MHS, Lam NKT, Sin HMY, Louis LHT (2014) Wrestling and Health In S. FONG (Ed.), Martial Arts for Health: Translating Research into Practice. OMICS Group Incorporation, USA, CA.

61. Lee KY, Lee PH, Macfarlane D (2014) Associations between moderate-tovigorous physical activity and neighbourhood recreational facilities: The features of the facilities matter. Int J Environ Res Public Health 11: 12594 12610.

62. Li EJ, Lam MHS, Louie LHT, Li SSS (2012) An analysis on history and cultural background of Chinese Tai Chi soft ball. Asian Journal of Physical Education and Recreation 18: 27-30.

63. Lau PW, Lam MHS, Leung BW (2010) National Identity and the Beijing Olympics: School Children's Responses in Mainland China, Taiwan and Hong Kong. Procedia Soc Behav Sci 2: 6729-6738.

64. Lau PW, Lam MHS, Leung BW, Choi CR, Ransdell LB (2012) The longitudinal changes of national identity in mainland China, Hong Kong and Taiwan before, during and after the 2008 Beijing Olympics Games. The International Journal of the History of Sport 29: 1281-1294.

65. Lau PW, Lam MHS, Leung BW (2011) The Beijing Olympics and Expressions of National Identity in China, Taiwan and Hong Kong. The Olympics in East Asia: Nationalism, Regionalism and Globalism on the Center Stage of World Sports, p: 147

66. Ho G, Yiu EYM, Lam MHS (2016) The Hong Kong games in the eyes of local sports and recreation students. The International Journal of the History of Sport 33: $1209-1225$.

67. Lam MHS (2010) Management Evaluation of the Healthy Athlete program of 2007 Shanghai Special Olympics World Summer Games. Asian Journal of Physical Education and Recreation 16.

\section{OMICS International: Open Access Publication Benefits \&}

\section{Features}

\section{Unique features:}

Increased global visibility of articles through worldwide distribution and indexing

Showcasing recent research output in a timely and updated maner

Special issues on the current trends of scientific research

Special features:

$700+$ Open Access Journal

$50,000+$ editorial team

Rapid review process

Quality and quick editorial, review and publication processing

Indexing at major indexing services

Sharing Option: Social Networking Enabled

Authors, Reviewers and Editors rewarded with online Scientific Credits

Better discount for your subsequent articles

Submit your manuscript at: http://www.omicsonline.org/submission/ 